powers. No doubt, new studies will utilise the revised version of the Geneva model. As for the Wells score used in our study, we think that neither dichotomised nor trichotomised scores make too much sense if they are combined with a D-dimer test. It must be noted that positive D-dimer test allows the shifting of some patients from the "unlikely" PE arm of the dichotomised Wells score back to the "likely" arm. Since only few COPD studies related to the Wells scoring system exist in the literature, we preferred including all COPD patients for evaluation who are considered naturally at risk for PE, as mentioned above, to excluding some unlikely PE patients with negative D-dimer test results. We believe this approach would be necessary for the further validation of the Wells system in this specific group of patients.

As a complicating or triggering factor, presence of VTE in COPD patients on exacerbation is an important issue. 1-yr mortality was found doubled in VTE cases in our study. We believe that clinical prediction rules developed for PE will help us to manage COPD. As L. Bertoletti and M. Righini also underline, in order to have better prediction powers in cases with severe underlying specific diseases, we might need some modifications in the current prediction models, or some new disease-specific models should be developed in future.

\section{H. Gunen*, G. Gulbas", E. In", O. Yetkin" and}

S.S. Hacievliyagil ${ }^{\#}$

*Division of Pulmonary Medicine, Umraniye Research and Training Hospital, Istanbul, "Division of Pulmonary Medicine, Turgut Ozal Research Center, Inonu University, and "Division of Pulmonary Medicine, Sevgi Private Hospital, Malatya, Turkey.

Correspondence: G. Gulbas, Division of Pulmonary Medicine, Turgut Ozal Research Center, Inonu University, Malatya 44069, Turkey. E-mail: gazigulbas@yahoo.com

Statement of Interest: None declared.

\section{REFERENCES}

1 Gunen $\mathrm{H}$, Gulbas G, In E, et al. Venous thromboemboli and exacerbations of COPD. Eur Respir J 2010; 35: 1243-1248.

2 LR Gregoire, Righini M, Roy PM, et al. Prediction of the pulmonary embolism in the emergency department: the revised Geneva score. Ann Intern Med 2006; 144: 165-171.

3 Torbicki A, Perrier A, Konstantinides S, et al. Guidelines on the diagnosis and management of acute pulmonary embolism: the Task Force for the Diagnosis and Management of Acute Pulmonary Embolism of the European Society of Cardiology (ESC). Eur Heart $J$ 2008; 29: 2276-2315.

4 Wolf SJ, McCubbin TR, Feldhaus KM, et al. Prospective validation of Wells criteria in the evaluation of patients with suspected pulmonary embolism. Ann Emerg Med 2004; 44: 503-510.

5 Runyon MS, Webb WB, Jones AE, et al. Comparison of the unstructured clinician estimate of pretest probability for pulmonary embolism to the Canadian score and the Charlotte rule: a prospective observational study. Acad Emerg Med 2005; 12: 587-593.

6 Rodger MA, Maser E, Stiell I, et al. The interobserver reliability of pretest probability assessment in patients with suspected pulmonary embolism. Thromb Res 2005; 116: 101-107.

\title{
Confirmation of asthma diagnosis in the community
}

\section{To the Editors:}

The study of LUKS et al. [1] highlights an area of asthma care that has important patient and economic implications. The authors strive to answer a very relevant clinical question related to practical clinical considerations surrounding asthma diagnosis: how many steps of a diagnostic algorithm are required to confirm diagnosis of asthma among patients previously diagnosed with asthma in the community? The authors demonstrate that $>90 \%$ of patients were confirmed with only one or two study visits by either pre- and postbronchodilator spirometry or a single bronchial challenge test. Based on the protocol design, the patients studied at visit 1 and visit 2 were similar, since steroid tapering did not occur until visit 3. From figure 1 in [1], it appears that 54 out of 499 (10.8\%) patients were diagnosed with asthma using simple pre- and post-bronchodilator spirometry. At visit 2, methacholine challenge testing resulted in a confirmation rate of 274 out of $444(61.7 \%)$ patients and an exclusion rate of 121 out of 444 $(27.3 \%)$ patients. In order to identify the most simple and practical approach to asthma diagnosis confirmation in this population, a methacholine challenge test (MCT) should have been performed at visit 1 . It is possible that some, if not most, of the spirometrically confirmed cases (visit 1) would also be confirmed with MCT. This issue is relevant because it would provide practical information about which test should be ordered first in the real world; the results of the study by LUKS et al. [1] suggest that MCT may be the option of choice among this population. If primary care physicians are to be encouraged to adopt a role in confirmation of asthma diagnosis in the community, this issue requires further clarification. The design of the current algorithm may actually underestimate its utility in terms of the number of visits required to confirm asthma diagnosis; this may turn out to be a one-visit process for most patients.

Given the algorithm design, it would be more clear to state that at least two visits were required to confirm or exclude a diagnosis of asthma in the majority of patients. Further studies are needed to determine how simple spirometry compares to MCT for de novo asthma diagnosis in the community setting. 


\section{A. D’Urzo}

Family and Community Medicine, University of Toronto, Toronto, Canada.

Correspondence: A. D’Urzo, Family and Community Medicine, University of Toronto, 1670 Dufferin Street, Suite, 107, Toronto, Ontario M6H 3M2, Canada. E-mail: tonydurzo@sympatico.ca

Statement of Interest: A statement of interest for A. D'Urzo can be found at www.erj.ersjournals.com/site/misc/statements.xhtml

\section{REFERENCES}

1 Luks VP, Vandemheen KL, Aaron SD. Confirmation of asthma in an era of over diagnosis. Eur Respir J 2010; 36: 255-260.

DOI: $10.1183 / 09031936.00136210$

\section{To the Editors:}

The study by LUKS et al. [1] published in the August 2010 issue of the European Respiratory Journal (ERJ) evaluates a diagnostic algorithm to confirm previous physician-diagnosed asthma, and concludes that pre- and post-bronchodilator spirometry or a single methacholine challenge test (MCT) is sufficient to confirm the diagnosis in $>90 \%$ of patients. In the remaining patients, a repeat MCT after partial or complete drug withdrawal is confirmatory. The study was prompted by the epidemiological trends showing increasing prevalence of asthma, raising the question of a real increase versus an overdiagnosis. Correct diagnosis is extremely important issue, as a correct estimate of the burden of disease has major medical, social and economic implications. The study has also received editorial comments by CONTOLI and PAPI [2] in the same issue of the ERJ.

There is an inherent assumption in the study that pre- and post-bronchodilator spirometry or MCT are gold standards for diagnosis of asthma, and those cases failing these tests can be labeled as "overdiagnosed". The criteria of an improvement in forced expiratory volume in $1 \mathrm{~s}$ (FEV1) of $\geqslant 200 \mathrm{~mL}$ and $\geqslant 15 \%$ after bronchodilator, used by the authors, lacks consensus. In fact, there are several debatable issues regarding the test of bronchodilator responsiveness, which is the best parameter to assess the response (forced vital capacity (FVC) or FEV1); what is a positive threshold of change $(12,15$ or $20 \%$ ) and what is the best method of expressing the result (percentage change over baseline or change as a percentage of predicted) [3]? The recommendations have varied from time to time and from country to country, and the recent consensus statement of the joint American Thoracic Society (ATS)/European Respiratory Society (ERS) task force considers an increase in FEV1 or FVC of $12 \%$ and $200 \mathrm{~mL}$ over the baseline as an indicator of responsiveness [4]. We have previously shown that bronchodilator responsiveness has limited application as a gold standard diagnostic test for asthma, as none of the above methods of expression of the response have adequate positive predictive value [5]. Patients with chronic obstructive pulmonary disease (COPD) are likely to meet the aforementioned criteria in nearly $20 \%$ of cases.

However, an MCT also has deficiencies as a confirmatory test. A provocative concentration of methacholine causing a $20 \%$ fall in FEV1 of $<8 \mathrm{~g} \cdot \mathrm{mL}^{-1}$ lacks adequate specificity [2] and false positive results are often seen in patients with other diseases, including COPD, allergic rhinitis and sarcoidosis. In their editorial comment on the article, CONTOLI and PAPI [2] have referred to limitations in its sensitivity and specificity that result in both false positive and negative results. This undermines its value as a diagnostic test.

The basic premise of the study is thus flawed and the conclusions based on this assumption would also be erroneous. Both the tests introduce a substantial dimension of over- and underdiagnosis and, hence, are not appropriate tools to evaluate a previous diagnosis.

Overdiagnosis may result from a lack of availability or choice of diagnostic methods, or an incorrect application and interpretation of these. The title of the article refers to "an era of overdiagnosis". However, in the evolution of epidemiological trends in asthma, the contribution of underdiagnosis is equally important. Several studies have documented the phenomenon of the iceberg of asthma morbidity pointing towards substantial underdiagnosis in asthma, which is also often diagnosed late in its course [6,7]. The study by LUKS et al. [1] was not designed to look into the underdiagnosis of asthma. An "era of misdiagnosis" may be a more apt phrase for the title than "an era of overdiagnosis".

While appreciating the study by LUKS et al. [1], CONTOLI and PAPI [2] have remarked that the suggested algorithm to reduce overdiagnosis of asthma is valuable but perfectible, and needs to be improved in order to better identify truly negative results. We believe that in the absence of a gold standard for diagnosis, a weighted, multidimensional diagnostic scoring system using multiple tools, including history, therapeutic responsiveness to drugs and objective tests, such as pre- and post-bronchodilator spirometry, MCT or peak flow variability, needs to be developed that can be adapted and applied at all levels of healthcare, from primary to tertiary. This would allow an evaluation of both over- and underdiagnosis in the epidemiological trends. We are still short of attaining perfection in the diagnosis of asthma in epidemiological studies or in clinical practice.

\section{S.K. Chhabra and M. Gupta}

Viswanathan Chest Hospital, Vallabhbhai Patel Chest Institute, University of Delhi, Delhi, India.

Correspondence: S.K. Chhabra, Department of Cardiorespiratory Physiology, Viswanathan Chest Hospital, Vallabhbhai Patel Chest Institute, University of Delhi, Delhi 110007, India. E-mail: skchhabra@mailcity.com

Statement of Interest: None declared. 